\title{
A COMPARISON OF GUIDED AND UNGUIDED ANTI-MISSILE KINETIC KILL
}

\section{COUNTERMEASURES}

\author{
Capt Mark C. Cherry \\ Flight Integration Branch Wright \\ Aeronautical Laboratories \\ Wright-Patterson AFB OH 45433- \\ 7505
}

\author{
LtCol Stuart C. Kramer, PhD \\ Dept of Aeronautics and Astronautics \\ Air Force Institute of Technology \\ Wright-Patterson AFB OH 45433- \\ 7765
}

\author{
Capt Joel J. Hagan \\ Space Experiment Directorate \\ Phillips Laboratory \\ Kirtland AFB NM 87109
}

\begin{abstract}
Modern Surface to Air Missiles (SAMs) present a significant threat to today's military and civilian aircraft. Current countermeasure systems such as flares and chaff rely on decoying the missile threat and do not provide adequate protection against advanced computerized missiles. An aircraft defense system that actively seeks out and defeats an incoming missile by placing a physical barrier in the missile's path offers a promising alternative to current countermeasures technology. This paper reports the results of a comparison study between self guided or "smart" missiles, which seek out SAMs, and unguided, or "dumb," countermeasures that are placed in the path of the incoming missile. Both systems utilize a kinetic kill mechanism and are designed to protect the aircraft from shoulder launched missiles while the aircraft is in the takeoff and climb-out configurations.
\end{abstract}

\section{Background}

Today's aircraft, both military and civilian, face increasing risk on any given flight. Crowded flight lanes, airports, and bird strikes increase the danger of losing an aircraft. In recent years, the shoulder launched surface to air missile has become an added threat to aircraft. As it has become the weapon of choice for many terrorists. Increasing costs of new aircraft for both the military and civilian purchasers have increased the need for selfprotection of these very valuable resources. The ability of terrorist and paramilitary groups to obtain shoulder launched surface to air missiles increases the risk to these high dollar assets from low cost SAMs. Being attacked by low cost surface to air missiles is a definite concern of the United States Air Force (USAF) and considerable research is being pursued in the areas of self-defense and automatic defense of aircraft. Modern Surface to Air Missiles (SAMs) present a significant threat to today's military and civilian aircraft. Current countermeasure systems such as flares and chaff rely on decoying the missile threat and do not provide adequate protection against advanced computerized missiles (Schaffer, 1993:1).

\section{Problem}

Currently investigators are researching the feasibility of using actively guided or "smart" expendables to place a barrier between the aircraft and the missile threat. Unguided or "dumb" countermeasures may be able to provide an equal protection capability at a reduced cost, and by employing less sophisticated technology. The tradeoff of employing a "dumb" kinetic kill device as opposed to a "smart" device is described in this paper.

In order to compare the two types of systems, a candidate smart device and a candidate dumb device were analyzed with respect to a common airframe and scenario. The $\mathrm{C} / \mathrm{KC}-135$ aircraft was chosen as the airframe because of its importance to the U.S. Air Force. It is one of the 
U.S. Air Force's most extensively used airframes, and is considered a force-multiplier aircraft. If shot down, the effectiveness of the aircraft it refuels or supports is significantly diminished. The scope of the comparison study was further narrowed by only considering the employment of the two defense systems in the takeoff and climb-out configurations. This scenario was chosen based on the extreme vulnerability of the aircraft during these phases of flight. The $\mathrm{C} / \mathrm{KC}-135$ has little excess power with which to maneuver and little time to react. It is assumed in this scenario that the airfield from which the $\mathrm{C} / \mathrm{KC}-135$ is taking off is secured, but is still vulnerable to terrorist attack by small groups. This scenario is similar to the recent humanitarian relief operation in Somalia.

Based on past experiences and background research conducted into active aircraft defense systems, eight characteristics deemed to be the most relevant to operational aircraft were used to compare smart and dumb active defense systems: the effectiveness of each kinetic kill device, the environmental impact of each device, the impact on both the aircraft and the mission of the aircraft, the installation requirements for each type of device, the life cycle cost, the maintenance requirements, and finally the amount of operator tasking required to operate each device. These eight areas are listed in Table 1 and will be later used to compare and contrast the relative value of smart and dumb active aircraft defense systems.

Table 1. Evaluation Areas

\begin{tabular}{|l|l|}
\hline Effectiveness & Environmental \\
Installation Reqmts & Cost \\
Impact on AVC & Impact on Mission \\
Maintenance & Operator Tasking \\
\hline
\end{tabular}

Threat

The real-world SAM used to model the threat SAM is the U.S. Army's Stinger missile. The Stinger is a shoulder launched surface to air missile employing passive infra-red homing which has all aspect engagement capability, meaning that it can fire at a target at any angle. It has a length of $1.5 \mathrm{~m}(5 \mathrm{ft})$, a weight of $98 \mathrm{~N} \mathrm{(22} \mathrm{lbf),} \mathrm{and} \mathrm{a} \mathrm{frontal}$ area of $39 \mathrm{~cm}^{2}\left(6 \mathrm{in}^{2}\right)$. Its performance parameters include a ceiling of $3000 \mathrm{~m}(9840 \mathrm{ft})$, a maximum range of $5 \mathrm{~km}$ ( 3 miles), and a top speed of $700 \mathrm{~m} / \mathrm{s}$ (2297 ft/sec) (Vulcan, 1990:208). The velocity profile used in this model is based on unclassified information provided by Wright Laboratory, and is used to characterize missile trajectory during flight (Voegle, 1995). The Stinger constantly spins at a frequency of 15 hertz and arms itself $2 \mathrm{~m}(6 \mathrm{ft})$ outside the launch tube (Voegle, 1995). The Stinger employs a contact fuse which is activated by a deceleration of $1226 \mathrm{~m} / \mathrm{s} 2(125 \mathrm{~g}$ 's), with a delay time between 0.3 and 0.6 microseconds (Doherty, 1995). Clearly this SAM is a formidable threat to aircraft landing or taking off at airfields.

\section{Candidate Systems}

As mentioned earlier, the purpose of this paper is to compare smart and dumb active defense systems for $\mathrm{C} / \mathrm{KC}-135$ aircraft in the take-off and climb-out scenario. The candidate smart system is a concept under development by TRW ${ }^{\top M}$ called The Aircraft Close In Defense System (ACIDS) (Stoddard, 1995). The candidate dumb system is a deployable net made of Detonation Chord ${ }^{\top M}$ (Det Net). It is based on research completed by a systems engineering design group at the Air Force Institute of Technology (AFIT) (Cherry et al, 1995). Each of the systems consists of three subsystems: detection and tracking, launcher, and expendable. The differences are primarily in the capabilities, complexity and cost of the expendable.

ACIDS, the candidate smart system, is built around a self-propelled, self-guided interceptor missile with a proximity fused warhead. Detection and tracking is accomplished with a millimeter wave radar system, which is also used to illuminate the threat missile for end-game engagement. The missile is launched from a pod system which can accommodate up to eight missiles. Each ACIDS missile weighs approximately $66.75 \mathrm{~N}$ (15 lbf) including a $22.25 \mathrm{~N}$ ( $5 \mathrm{lbf}$ ) warhead. The on-board missile controller uses a millimeter wave homing receiver to give semi-active guidance during the intercept. Since it is able to steer in flight toward the threat, it is considered a smart system. The 
entire system is automatic so that it can react quickly to threats, although the crew can switch the system on and off. It is designed for minimal interaction with the aircraft but does need aircraft power and access to the aircraft data bus.

The Det Net was used to evaluate dumb candidate systems. The Det Net's detection and tracking system consisted of a detection system based on a composite of two commercial off-theshelf (COTS) detection systems, the AAR-47 and the ALQ-156. Both of these systems are quadrant detectors, with no range information. The tracker used for the Det Net is also a composite of two COTS trackers, the AAR-44 and the AAR-54. In addition a ranging antenna is also on the expendable itself. The Det Net is launched by a lazy susan device that is similar to the ALE-17 flare buckets currently employed on AC-130 aircraft. The expendable subsystem consists of a hollow bullet shaped canister, with a $6.5 \mathrm{~cm}$ (2.56 in) radius and a $39 \mathrm{~cm}$ (15.4 in) length containing a 14 sided polygonal net made of Detonation Chord ${ }^{\mathrm{TM}}$. Based on range information from the tracker, the system launches the expendable at the proper time, inclination, and azimuth to intercept the expected trajectory of the threat. At the appropriate time, the canister ejects the net which expands to block the threat. The net is weighted at its periphery so that it expands evenly and achieves stable flight for a few seconds. When the threat missile impacts the net, it shears the detonation chord and detonates the entire net. Since the flight of the canister is ballistic and includes no terminal guidance, the expendable is considered dumb.

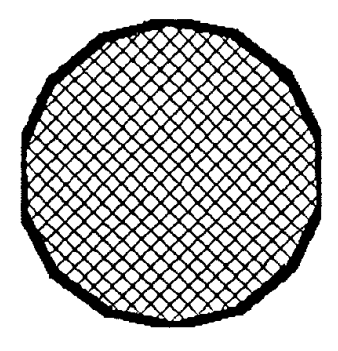

Figure 1. Det Net Deployed Geometry
The ACIDS concept system has the ability to intercept SAM threats at various distances from the aircraft. The Det Net concept requires that the system is deployed to intercept the SAM threat at a set range from the aircraft. This requirement allows the Det Net system to concentrate on a smaller area to intercept threats. In the stated scenario, the difference between the intercept distances for the two systems was not significant. Different scenarios may drive different effectiveness results for the two systems.

\section{Evaluation}

This section gives a qualitative summary of results of the evaluation, for a complete detailed discussion, the reader is referred to the design report (Cherry, et al, 1995). In order to make a comprehensive comparison of the two types of systems, an evaluation was made between ACIDS and the Det Net using the eight different areas listed earlier. The importance of each of these areas may vary between decision makers, but they are all important factors that must be evaluated to determine the best solution. Models for each of these eight areas were developed by the design group and used to evaluate the ACIDS and Det Net systems. Each of the models produced a score between 1 and 100 for the two systems. The score offered an easy way to make comparisons between the two systems. The score also offered a means to incorporate the relative importance of one area versus any other area, in order to make a system comparison.

Effectiveness is a measure of how well each aircraft defense system is able to eliminate the SAM threat. From the pilot's point of view this is the most important characteristic of a system, since the pilot will be put in harms way. Effectiveness is characterized in terms of three parameters: probability of kill, passivity, and false alarm rate. Probability of kill is the percentage of the time that the system will successfully intercept the threat and prevent it from reaching the aircraft. Passivity rates the level of electromagnetic radiation emitted by the aircraft's detection and tracking systems. The false alarm rate evaluates how often the aircraft 
defense system identifies a false threat. The ACIDS system rated slightly below the Det Net in all three effectiveness evaluations. The area that a deployed ACIDS missile can cover at any one time is much less than the area covered by a deployed net device. What the Det Net lacked in accuracy due to a COTS tracking system and no terminal guidance, it made up for in the amount of area covered by the deployed system. The edge in effectiveness between smart and dumb systems lies slightly with the Det net.

Environmental Impact evaluates how the aircraft defense system will adversely affect humans, animals, and plant life. Environmental safety is a growing concern with all new weapons systems developed by the U.S. Air Force. The impact area and terminal velocities of the deployed systems are used to calculate the amount of kinetic energy the expendables impart on the earth. This energy, combined with the temperature at impact, help to determine the potential for damage to humans and property from the falling deployed system and SAM fragments. When the systems were compared on an environmental basis, the ACIDS system came out on top. The projected amount of debris and collateral ground damage from a successful ACIDS intercept was less than that from the Det Net. The reason for the difference is that the Det Net is deployed in separate pieces. The canister used to house the net will fall to the ground along with the missile and net fragments. ACIDS scores on top for environmental concerns.

Impact on Aircraft is based on how much of the aircraft's resources such as space (volume), load carrying capability, and electrical power are consumed by the aircraft defense system. If the system requires excessive amounts of a certain type of resource, it could seriously degrade the flexibility of the aircraft, and may not be worth installing. Both systems would have a significant impact on a $\mathrm{C} / \mathrm{KC}-135$ aircraft. Any time a new system is added to an aircraft many tests have to be accomplished to ensure flight control is not adversely affected. The ACIDS system, however, had less impact on the aircraft than the Det Net system. Both systems had similar weight, volume, and power requirements, but the center of gravity affects were deemed to be much less for the ACIDS system. The podded ACIDS system can be placed in a variety of positions to adapt to the stability needs of the aircraft. The Det Net system must be placed on the aircraft in such a manner that it can be swiveled to the SAM threat area and so has much less positional flexibility.

Operator Tasking refers to how much time and energy must be expended by the flight crew in order to operate this aircraft defense system. If the system consumes large amounts of the flight crew's precious time and energy, it may be considered infeasible. Both the ACIDS, and Det Net systems required little operator intervention. Both systems work in an autonomous mode, and only need to be turned on and off by the pilot.

Monetary Cost refers to the entire life cycle cost of the system, from initial requirements definition to system disposal. Clearly, the greater the life cycle cost, the lower the desirability of the aircraft defense system. This area of evaluation had the greatest range of speculation. The Det Net detection and tracking system is expected to be less costly to develop and acquire since it is based on COTS equipment. The ACIDS launcher should be less expensive since it is much less complex. The Det Net expendable should be much less expensive because it is very simple and does not contain any active components. The difference in expendable costs dominate since we expect to purchase and support many expendables for each launcher system. Overall, the Det Net was judged to be much less expensive than the ACIDS.

Impact on Mission is defined in terms of performance degradation such as reductions in range and endurance resulting from the aircraft defense system. Excessive performance degradation would significantly reduce the utility of the aircraft defense system. Neither the Det Net nor the ACIDS systems had significant impact on the primary mission of the $\mathrm{C} / \mathrm{KC}-135$ aircraft. The installed systems would not adversely effect the aircraft getting to its target altitude or location and delivering fuel. Both systems can be manually turned on and off, which mitigates the possibility of 
an unwanted deployment during a refueling mission.

Installation Requirements characterize the amount of effort required to modify the aircraft and install the aircraft defense system. Even if a system is effective and inexpensive, for example, if it takes a great deal of time and personnel to install it, the system may not be considered worth the effort.

The ACIDS system required the least amount of modification support from an Air Logistics Center (ALC) or a maintenance depot. Both systems require a significant amount of change to internal radar and data bus interfaces. However, the ACIDS pod system may be mounted externally on wing or fuselage, while the Det Net launcher must be firmly mounted on the aircraft frame.

The Air Force places a significant emphasis on the maintenance requirements of new weapons systems. The ease of maintenance is of primary importance to effective day-to-day operation of the aircraft. If the aircraft spends most of its life on the ground in order to perform maintenance on the aircraft defense system, there may not be a need for a defense system to protect the aircraft for the short period of time that it is in the air. The maintenance requirements for both the ACIDS and Det Net systems were determined to be similar. Neither system requires a significant increase in weekly flight line maintenance. Conceptually, each system offers external subsystem and system checks. These checks allow the maintenance crews to isolate and remove problem subsystems, without removing the entire system.

Overall, the Det Net is superior in cost and effectiveness while ACIDS is best in environmental impact, impact on aircraft, and installation requirements. For a broad range of possible relative weights on each of these areas, the Det Net received the top composite score and was the favored solution. Table 2 illustrates the results of the evaluation.
Table 2. Evaluation Results

\begin{tabular}{|l|c|l|l|l|}
\hline & Effect. & Environ. & Imp AC & Imp Mis \\
\hline Det Net & $\mathrm{X}$ & & & \\
\hline Tie & & & & $\mathrm{X}$ \\
\hline ACIDS & & $\mathrm{X}$ & $\mathrm{X}$ & \\
\hline & Inst. Req & Cost & Main & Op Task \\
\hline Det Net & & $\mathrm{X}$ & & \\
\hline Tie & & & $\mathrm{X}$ & $\mathrm{X}$ \\
\hline ACIDS & $\mathrm{X}$ & & & \\
\hline
\end{tabular}

\section{Conclusion}

In comparing the two candidate systems, the importance placed on each evaluation area became the key to determining the best type of system for the given scenario and aircraft. The two systems did not offer a significant difference when evaluated under operator tasking, impact on mission, or maintenance. The differences came when the two systems were evaluated under the areas of cost, effectiveness, environmental impact, impact on aircraft, and installation requirements.

The ACIDS system concept was the clear choice when evaluated under environmental concerns, impact on aircraft, and installation requirements. However, the Det Net system had the edge for effectiveness and cost. The effectiveness and cost of a SAM protection device is of paramount concern to a potential system user. If new systems cannot do the job they are designed to do well, they are of no use. A user demands confidence that a SAM protection system will offer a return on investment by saving an aircraft and air crew from destruction. For this reason, the Det Net, or dumb, candidate system was determined to be favorable over the smart, ACIDS system.

Today's threat environment is not significantly different from today's monetary environment. A return on investment is the key decision driver for developing new systems, or building new airports. If a system, no matter how state of the art, cannot show that it takes care of a need cheaply, and effectively, it will not be developed. Other evaluation areas serve as a way to discriminate between systems that are similar in effectiveness, and cost. The Det Net system satisfies today's requirements better than the ACIDS systems concept. 


\section{Bibliography}

Cherry, M. C. Dewitt, B. R. Dusseault, C. G. Hagan, J. J. Peterson, B. S. Development of an Active Air Defense System for the $\mathrm{C} / \mathrm{KC}-135$ Aircraft. MS thesis, AFIT/GSE/ENY/95D-01. School of Engineering, Air Force Institute of Technology (AU) Wright-Patterson AFB OH, December 1995.

Doherty, Robert. Missile Analyst, Missile and Space Intelligence Command, Huntsville AL. Telephone interview. 20 July 1995.

Schaffer, Marvin B. Concerns About Terrorist With Manportable SAMS. Santa Monica CA: Rand Corporation, 1993.

Stoddard, Frank J. Program Manager, Propulsion \& Combustion Center, TRW

Space \& Technology Division, Space \& Electronics Group, Redondo Beach CA.

Telephone interview. 12 May - 13 Oct 1995.

Voegle, George. Electrical Engineer, Wright Laboratory, Electronic Warfare Division, WrightPatterson AFB OH. Personal interview. 16 May 1995.

"Vulcan Air Defense System" Jane's Weapon Systems. Alexandria VA: Jane's Information Group, 1989-1990. 